\title{
Diagnostic value of paraclinical tests in multiple sclerosis: relative sensitivities and specificities for reclassification according to the Poser committee criteria
}

\author{
S Beer, K M Rösler, C W Hess
}

\begin{abstract}
The yield of paraclinical tests was evaluated in a prospective study of 189 consecutive patients referred for suspected multiple sclerosis (142 patients with multiple sclerosis, 47 non-multiple sclerosis patients on discharge). Patients were first classified according to the Poser criteria by the clinical findings. Subsequently, the results of paraclinical tests (cranial MRI, visually evoked potentials (VEPs), somatosensory evoked potentials by tibial nerve stimulation (SSEPs), motor evoked potentials (MEPs), and analysis of CSF for oligoclonal banding and IgG-index (CSF)) were taken into account. The percentage of reclassified patients (reclassification sensitivity, RS) was always lower than the percentage of abnormal results (diagnostic sensitivity, DS), and the divergence of RS $v$ DS differed between the tests $(60 \% v 84 \%$ in MRI, $31 \% v 77 \%$ in CSF, $29 \% v 37 \%$ in VEPs, $20 \% v 68 \%$ in MEPs, and $12 \% v 46 \%$ in SSEPs respectively). False reclassifications of nonmultiple sclerosis patients to multiple sclerosis would have occurred with all tests (MRI: six of 47 patients, (reclassification specificity $88 \%$ ); CSF: one $(98 \%)$; VEPs: two (96\%); MEPs: two (96\%); SSEPs: four (91\%); $P<0.05)$. Although MRI had superior diagnostic capacity, 57 of the 142 patients with multiple sclerosis were not reclassified by the MRI result, 12 of whom were reclassified by CSF and 18 by one of the evoked potential (EP) studies. Of the 98 patients not reclassified by CSF, 53 were reclassified by MRI and 39 by EPs. The results suggest that for the evaluation of paraclinical tests in suspected multiple sclerosis, comparison of diagnostic sensitivities is inappropriate. In general, a cranial MRI contributes most to the diagnosis; however, due to its comparatively low specificity and its considerable number of negative results, EP or CSF studies are often useful to establish the diagnosis of multiple sclerosis.
\end{abstract}

$(\mathcal{F}$ Neurol Neurosurg Psychiatry 1995;59:152-159)

Keywords: multiple sclerosis; evoked potentials; magnetic brain stimulation; magnetic resonance imaging

The diagnosis of multiple sclerosis is based on the detection of multiple inflammatory demyelinating white matter lesions, which are disseminated in time and space. ${ }^{12}$ Clinically, dissemination in time is assessed by analysing the course of the disease or by serial examinations, and dissemination in space is suspected if clinical findings cannot be explained on the basis of a single lesion. In many patients, however, the clinical assessment is not sufficient to prove temporal and spatial dissemination, and paraclinical studies have to be performed. Clinically asymptomatic lesions may be detected by evoked potential studies or MRI of the brain and spinal cord. In addition, CSF analysis may provide evidence for the inflammatory nature of the disease. None of the clinical or paraclinical findings are, however, specific for the diagnosis of multiple sclerosis. Early classification criteria were based on clinical data only. ${ }^{1}$ The Poser Committee Criteria (PCC), published in 1983, include paraclinical tests as aids for the diagnostic classification of multiple sclerosis. ${ }^{2}$ Since then, newer techniques such as MRI and motor evoked potentials (MEPs) have been developed.

The aim of the present investigation was to analyse the diagnostic yield of paraclinical tests in multiple sclerosis. Most previous investigations on this topic analysed the frequency of an abnormal result in a given test ("sensitivity") in patients with multiple sclerosis, ${ }^{3-9}$ yet high sensitivity of a test in multiple sclerosis does not equal high diagnostic yield. For instance, a paraclinical test that tends to confirm clinically detectable signs is not very likely to improve the diagnostic certainty in a patient, even though it might be very sensitive in terms of yielding a high proportion of abnormal results. Only a diagnostic test which, by the nature of its method and the neural system assessed, is apt to detect clinically silent lesions will be powerful in increasing diagnostic certainty. Thus a different approach is necessary to compare the diagnostic impact of paraclinical investigations in multiple sclerosis. Only a few studies have used a stratified approach to assess the increase of diagnostic accuracy from a clinical baseline by adding stepwise information obtained by paraclinical investigations. ${ }^{1011}$ None of these included MEPs, even though the diagnostic sensitivity of MEPs in multiple sclerosis exceeds that of somatosensory evoked potentials (SEPs), visually evoked potentials (VEPs), and early acoustically evoked brainstem potentials. ${ }^{12-15}$ In this study, we analysed the number of patients with suspected multiple sclerosis who could be reclassified after the clinical examination 
according to the PCC by a given test result or by combining results of several tests. These paraclinical tests were: MEPs recorded from upper and lower limb muscles, SEPs by stimulation of the tibial nerve (SSEPs), VEPs, cranial MRI, and CSF analysis. By its design, the study included some patients with initially suspected multiple sclerosis, in whom subsequently a different diagnosis was made. Comparison of results in these non-multiple sclerosis patients with the results in patients with multiple sclerosis additionally provided a measure for the specificity of these paraclinical tests. Some aspects of this study have been published previously in a preliminary form. ${ }^{16}$

\section{Material and methods \\ PATIENTS}

We prospectively included all patients with suspected multiple sclerosis referred to our neurological department between 1989 and 1993. Only patients who agreed to be studied in hospital and who lacked a contraindication for one of the tests performed were included (presence of a cardiac pacemaker, history of epileptic fits or intracranial operations, clinical signs of increased intracranial pressure, age under 16 years). We did not include patients who presented with a clinically definite multiple sclerosis according to PCC because, by definition, paraclinical tests would not improve the diagnostic certainty in these patients. Patients with known diseases of the CNS other than multiple sclerosis were also excluded. All patients underwent a clinical examination, CSF analysis, cranial MRI, and multimodal evoked potentials (VEPs, SSEPs, MEPs).

Based on the diagnosis on discharge, we grouped the patients into non-multiple sclerosis patients (patients in whom a different diagnosis was established during the study), and patients with multiple sclerosis, comprising patients with definite, probable, and "possible" multiple sclerosis. Patients were classified as having possible multiple sclerosis if they did not meet the PCC criteria on discharge and no other CNS diseases were found, so that the suspicion of multiple sclerosis was maintained.

PARACLINICAL TESTS

All tests complied with the criteria of the local ethics committee.

CSF was evaluated for the presence of oligoclonal banding by silver staining after isoelectric focusing on polyacrylamide gel, ${ }^{17} 18$ and intrathecal IgG production ${ }^{19}$ indicating an inflammation of the CNS. Normal values given by Reiber ${ }^{20}$ were applied.

The VEPs and SSEPs were measured in a standardised manner, ${ }^{21}$ and the latency of the P100 (VEP) and N22/P40 (SSEP) components were compared with our own normative data. The criteria for VEP abnormalities were an absence of P100, a prolonged P100 latency, or an interocular difference of P100 amplitude and latency beyond the mean \pm 2.5 SD. Results for SSEPs were considered abnormal if the scalp recorded potential P40 was absent or if the central conduction time (N22-P40) or the side to side differences in latency and amplitude were beyond the mean $\pm 2.5 \mathrm{SD}$. We chose not to include early acoustic evoked brainstem potentials as their diagnostic value in multiple sclerosis has been shown to be limited. ${ }^{1015} 22-25$

The MEPs were recorded bilaterally from the brachial biceps, the abductor digiti minimi, and the anterior tibial muscle. Transcranial magnetic brain stimulation was performed with a custom made magnetic stimulator and a circular coil of $9 \mathrm{~cm}$ diameter. Stimulator and coil placement have been described previously. ${ }^{26}$ Results for MEPs were considered as abnormal if the cortically evoked potentials were absent, or if the central motor conduction time (CMCT) or the side to side differences in CMCT were beyond 2.5 SD of the mean.

Cranial MRI was usually performed with a 1.5 Tesla General Electric scanner. In some patients, MRI had been performed in other institutions before referral; in these instances, the scan was only repeated if the quality of the external examination did not meet our standards. In all studies, $\mathrm{T} 1$ and $\mathrm{T} 2$ weighted sequences were available, as well as axial, coronal, and sagittal slices.

\section{DIAGNOSTIC CLASSIFICATION}

For each patient a baseline PCC classification was established based on the clinical data only: clinically probable multiple sclerosis (C1, C2: abbreviations according to Poser et $a l^{2}$ ), and "possible" multiple sclerosis for patients not fulfilling the PCC. Patients with clinically definite multiple sclerosis (A1) where excluded for the reasons mentioned. Subsequently, the findings of each single paraclinical test and the results of various combinations of paraclinical tests were additionally taken into account and the patient was reclassified. These classifications were done by two of us (SB, KMR) independently, and in the few cases of disagreement were jointly discussed and classified. The reclassified patients were then counted for each test and each combination of tests.

From the clinical baseline classification, abnormal paraclinical test results were only considered for reclassification if they proved a separate lesion in a different functional system. In particular, MEP findings were only considered for reclassification if the clinical examination did not show brainstem dysfunction, pyramidal signs, or motor deficits of the respective spinal segment. For instance, if clinically a patient had a paraparesis with exaggerated tendon jerks of the legs, an abnormal MEP to the tibialis anterior was not considered for reclassification, but an abnormal MEP to the biceps was considered as evidence of a second lesion. Results for SSEPs were interpreted similarly-that is, only if there was no brainstem disturbance and no sensory deficit. Abnormal VEP findings were only considered for reclassification if no visual disturbance of the same side had influenced 
the baseline PCC classification. When the yield of multimodal EPs was assessed, an abnormal result for both MEPs and SEPs was not considered as evidence of two lesions if both could be attributed to the same spinal or brainstem location. Findings from MRI were taken into account in two different ways. Firstly, all MRIs demonstrating multiple areas of increased signal (multifocality) or single areas that could not account for the clinical symptoms of multiple sclerosis, were accepted for reclassification. Secondly, MRIs were only accepted as indicative of dissemination in space if they fulfilled the MRI criteria proposed by Fazekas et $a^{27}$ : three or more areas of increased signal and the presence of at least two of the following characteristics: $(a)$ lesion size $>5 \mathrm{~mm}$, (b) lesion abutting the ventricular bodies, and (c) infratentorial lesion location. These criteria had the greatest specificity for multiple sclerosis in a comparative analysis. ${ }^{28}$

\section{ANALYSIS OF RESULTS}

Five parameters were calculated for each paraclinical test. These were: (a) the diagnostic sensitivity, which was defined as the percentage of patients with an abnormal result in a given test or combination of tests; $(b)$ the reclassification sensitivity, which was defined as the percentage of patients with multiple sclerosis that could be reclassified according to PCC by results of a given test or a combination of tests; $(c)$ the reclassification index, which was defined as the ratio of reclassification sensitivity and diagnostic sensitivity; (d) the diagnostic specificity, which was defined as the percentage of non-multiple sclerosis patients with a normal finding in a given test; and (e) the reclassification specificity, which was defined as the percentage of non-multiple sclerosis patients that were correctly not reclassified by a given test. To assess significant differences of these parameters between the paraclinical tests, McNemar's ${ }^{-} 2$ test was applied. The level of significance was set at $\mathrm{P}=0.05$.

\section{Results}

PATIENTS

Inclusion criteria were fulfilled by 189 patients (107 women, 82 men). Their mean age was 38 (range 16-67) years, and the mean duration of the illness before admission to hospital was $2.9(0-25)$ years. These 189 patients were split into two groups based on the discharge diagnosis: the multiple sclerosis group consisted of 142 patients (80 women, 52 men), containing 98 patients with definite, 15 with probable, and 29 with possible multiple sclerosis. Of the 29 patients with possible multiple sclerosis, 14 had a chronic progressive course, and 15 had a first attack with consecutive remission. Of these 15 patients, nine experienced further bouts after a mean follow up of two years, allowing the later classification as probable or definite multiple sclerosis. Fifty five patients presented with a spinal cord syndrome, 40 had a brainstem symptomatology, 12 had an optic neuritis, and 35 had other CNS symptoms of multiple sclerosis. The mean age in the multiple sclerosis group was 37 (range 16-66) years. Table 1 shows the classification of the patients with multiple sclerosis to the diagnostic categories using the PCC and including all clinical and paraclinical data.

Forty seven patients ( 27 women, 20 men) had other neurological diseases or functional disturbances (non-multiple sclerosis group). Their mean age was 41 (range 19-67) years. The diagnoses in this group were psychogenic deficits (15), cerebrovascular diseases (13), non-inflammatory myelopathy (five), other inflammatory diseases of the CNS (four), and various other neurological diseases (10).

\section{DIAGNOSTIC AND RECLASSIFICATION} SENSITIVITY

Diagnostic sensitivities (percentage of abnormal results in the multiple sclerosis group) differed considerably between the paraclinical tests (table 2). The most sensitive tests to detect an abnormality were cranial MRI and CSF. The lowest diagnostic sensitivities were found for SSEPs and VEPs. As expected, the reclassification sensitivity (the percentage of patients reclassified by these abnormal test results) was generally lower than the diagnostic sensitivity. The reclassification sensitivity of MRI was greater than of all other examinations, whether the criteria of Fazekas et al ${ }^{27}$ were applied or not. It is noteworthy that all MRIs fulfilling the criteria of Fazekas et al ${ }^{27}$ led to a reclassification (reclassification index

Table 1 Classification of patients with multiple sclerosis $(n=142)$ based on the Poser committee criteria (PCC) before and after paraclinical testing

\begin{tabular}{|c|c|c|c|c|c|c|c|c|}
\hline \multicolumn{7}{|c|}{ Poser committee criteria for multiple sclerosis classification } & \multicolumn{2}{|l|}{ Our patients } \\
\hline \multicolumn{2}{|l|}{ Classification } & \multirow{2}{*}{$\frac{\text { Attacks }}{2}$} & \multicolumn{2}{|l|}{$\begin{array}{l}\text { Clinical } \\
\text { evidence }\end{array}$} & \multirow[t]{2}{*}{$\begin{array}{l}\text { Paraclinical } \\
\text { evidence }\end{array}$} & \multirow[t]{3}{*}{$C S F$} & \multirow[t]{2}{*}{$\begin{array}{l}\text { Baseline } \\
\text { classification } \\
\text { (n) }\end{array}$} & $\begin{array}{l}\text { Discharge } \\
\text { classification } \\
(n)\end{array}$ \\
\hline Clinically definite MS & A1 & & 2 & & & & & \\
\hline & $\mathrm{A} 2$ & 2 & 1 & and & 1 & & & 50 \\
\hline Laboratory supported & $\mathrm{B} 1$ & 2 & 1 & or & 1 & + & & 5 \\
\hline definite MS & B2 & 1 & 2 & & & + & & 1 \\
\hline & B3 & 1 & 1 & and & 1 & + & & 42 \\
\hline Clinically probable MS & $\mathrm{Cl}$ & 2 & 1 & & & & 57 & 4 \\
\hline & $\mathrm{C} 2$ & 1 & 2 & & & & 3 & 1 \\
\hline & $\mathrm{C} 3$ & 1 & 1 & and & 1 & & & 10 \\
\hline $\begin{array}{l}\text { Laboratory supported } \\
\text { probable MS } \\
\text { "Possible" MS }\end{array}$ & D1 & 2 & & & & + & 82 & 20 \\
\hline
\end{tabular}

Baseline classification from clinical data only; discharge classification based on clinical data and results of all paraclinical tests; MS $=$ multiple sclerosis; for definition of possible multiple sclerosis see text. 
Table 2. Number and frequency of abnormal findings (diagnostic sensitivity) and of reclassified patients (reclassification sensitivity) for the different paraclinical tests in the multiple sclerosis group $(n=142)$

\begin{tabular}{|c|c|c|c|}
\hline & $\begin{array}{l}\text { Diagnostic sensitivity } \\
\text { No abnormal findings (\%) }\end{array}$ & $\begin{array}{l}\text { Reclassification sensitivity } \\
\text { No patients reclassified (\%) }\end{array}$ & $\begin{array}{l}\text { Reclassification } \\
\text { index }\end{array}$ \\
\hline $\begin{array}{l}\text { MRI } \\
\text { MRI (Fazekas et } a l^{27} \text { ) } \\
\text { CSF } \\
\text { MEP } \\
\text { SSEP } \\
\text { VEP }\end{array}$ & $\left.\begin{array}{r}119(84) \\
85(60) \\
110(77) \\
96(68) \\
66(46) \\
53(37)\end{array}\right\} \quad P<0<0.005$ & 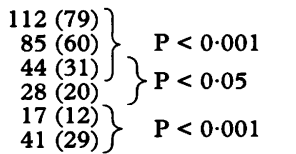 & $\begin{array}{l}0.94 \\
1.00 \\
0.40 \\
0.29 \\
0.26 \\
0.77\end{array}$ \\
\hline
\end{tabular}

The diagnostic impact of an abnormal result of a paraclinical test is expressed by the reclassification index (n reclassified/n abnor$\mathrm{mal}$ ). MRI results were interpreted with and without taking into account the criteria by Fazekas, et a ${ }^{7}$. Brackets indicate statistically significant differences (McNemar's $\chi^{2}$ test).

=1). The reclassification sensitivities of CSF and VEPs were lower than that of MRI $(P<$ 0.001 ), followed by MEPs and SSEPs (table 2). Compared with CSF, an abnormal finding in MRI and VEPs was of greater diagnostic value, as the diagnostic impact of an abnormal result, expressed by the reclassification index,

Reclassification to definite MS (

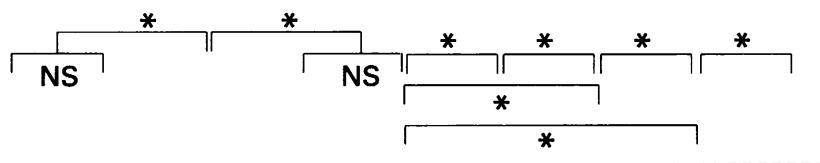

Reclassification to probable or definite MS (而):

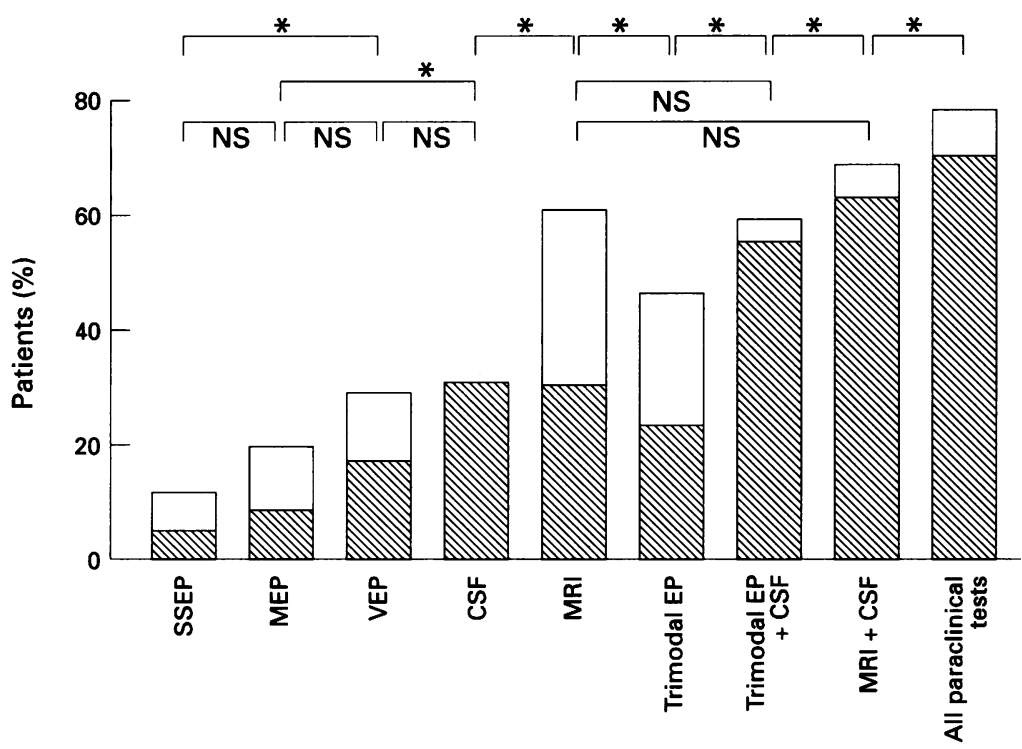

Reclassification sensitivity (percentage of reclassified patients) for each of the paraclinical tests alone and in combination (multiple sclerosis group, $n=142$ ). Hatched bars: reclassified to definite multiple sclerosis; open bars: reclassified to probable multiple sclerosis. Significances ( ${ }^{*} P<0.05 ;$ McNemar's $\chi^{2}$ test) are given separately for reclassification to definite and to probable or definite multiple sclerosis. MRI is interpreted according to Fazekas et al. ${ }^{27}$ was much greater (table 2). The low reclassification index of MEPs and SSEPs reflects the low yield of an abnormal result of these examinations for reclassification.

The figure shows the comparison of the reclassification sensitivity for single and combined tests. Because of the different appreciation within the PCC, CSF resulted in a much greater rate of reclassifications to definite multiple sclerosis than the other tests. The reclassification sensitivity of the cranial MRI alone was greater than that of multimodal EPs $(\mathrm{P}<$ 0.01). Only when multimodal EPs were combined with CSF results, did they reach the same reclassification sensitivity as MRI alone. With this combination, a greater number of patients were reclassified to definite multiple sclerosis than with MRI $(P<0.001)$.

Even though MRI was the superior test, the other tests were helpful in some aspects. Table 3 shows that about $20 \%$ of the patients with negative MRI could be reclassified by the other tests. As stated, CSF was particularly helpful for reclassification of patients to definite multiple sclerosis. Twelve of 57 patients with negative MRI (21\%) were reclassified by CSF findings and 18 of 57 (32\%) were reclassified by multimodal EPs. On the other hand, MRI findings allowed reclassification in 53 of 98 patients (54\%) who could not be reclassified by CSF results, and in 38 of 77 patients (49\%) not reclassified by multimodal EPs.

For MEPs, we analysed the reclassification sensitivity for the three recorded muscle pairs separately. There were no significant differences between the reclassification sensitivities of MEPs to these target muscles (brachial biceps $9 \cdot 2 \%$, abductor digiti minimi $10 \cdot 6 \%$, and anterior tibial muscle $10 \cdot 6 \%$ ). By combining the MEP results of two or three target muscles, the reclassification sensitivity increased significantly $(P<0.05)$.

Table 3 Patients in the multiple sclerosis group ( $n=142)$ that could not be reclassified by a given paraclinical test $(-)$ and patients that would have been reclassified by additional paraclinical tests $(+)$ if the initial single test was normal

\begin{tabular}{|c|c|c|c|c|c|c|c|}
\hline & $\begin{array}{l}\text { Not } \\
\text { reclassified }\end{array}$ & $\begin{array}{l}\text { MRI } \\
\left(\text { Fazekas et al }{ }^{27}\right)+\end{array}$ & $C S F+$ & $\begin{array}{l}\text { Multimodal } \\
E P+\end{array}$ & $M E P+$ & $S S E P+$ & $V E P+$ \\
\hline $\begin{array}{l}\text { MRI (Fazekas et } a^{27} \text { ) } \\
\text { CSF - } \\
\text { Multimodal EP - } \\
\text { MEP - } \\
\text { SSEP - } \\
\text { VEP - }\end{array}$ & $\begin{array}{r}57 \\
98 \\
77 \\
114 \\
125 \\
101\end{array}$ & $\begin{array}{l}\overline{53}(9) \\
38(17) \\
67(33) \\
73(37) \\
55(24)\end{array}$ & $\begin{array}{l}12(12) \\
\overline{18}(18) \\
35(35) \\
38(38) \\
25(25)\end{array}$ & $\begin{array}{l}18(9) \\
39(7) \\
-37(21) \\
48(26) \\
24(8)\end{array}$ & $\begin{array}{l}10(4) \\
19(3) \\
0(0) \\
\frac{23}{18(10)} \\
18(7)\end{array}$ & $\begin{array}{r}5(3) \\
11(1) \\
0(0) \\
12(5) \\
77(2)\end{array}$ & $\begin{array}{l}11(8) \\
22(6) \\
0(0) \\
31(20) \\
31(20) \\
-\end{array}$ \\
\hline
\end{tabular}

Number of patients that would have been reclassified to definite multiple sclerosis are in parentheses. MRI was always interpreted according to Fazekas et al. ${ }^{27}$ Multimodal EP = combination of MEPs, SSEPs, and VEPs. 
Table 4 Results for the 47 patients of the non-multiple sclerosis group

\begin{tabular}{|c|c|c|c|c|}
\hline & $\begin{array}{l}\text { No of abnormal } \\
\text { results (\%) (sensitivity) }\end{array}$ & No falsely reclassified by $P C C$ to $M S(\%)$ (diagnosis) & $\begin{array}{l}\text { Diagnostic } \\
\text { specificity (\%) }\end{array}$ & $\begin{array}{l}\text { Reclassification } \\
\text { specificity (\%) }\end{array}$ \\
\hline MRI & $18(38)$ & $\begin{array}{l}15 \text { (32) (8 CVI, } 1 \text { Lyme disease, } 1 \text { mitochondrial disease, } 1 \text { spinal } \\
\text { tumour, } 4 \text { functional deficits) }\end{array}$ & 62 & 68 \\
\hline MRI (Fazekas et $\left.a l^{27}\right)$ & $6(13)$ & 6 (13) (4 CVI, 1 Lyme disease, 1 mitochondrial disease) & 87 & 87 \\
\hline CSF & $7(15)$ & 1 (2) (1 unclear CNS inflammation) & 85 & 98 \\
\hline MEP & $11(23)$ & 2 (4) (1 spinocerebral atrophy, 1 thoracic myelopathy) & 77 & 96 \\
\hline SSEP & $7(15)$ & 4 (9) (1 CVI, 1 Lyme disease, 2 psychogenic) & 85 & 91 \\
\hline VEP & $5(11)$ & 2 (4) (1 CVI, 1 cervical myelopathy) & 89 & 96 \\
\hline
\end{tabular}

MS = multiple sclerosis; diagnostic specificity = percentage of correct negative results; reclassification specificity = percentage of correctly unreclassified patients. MRI was interpreted with criteria given by Fazekas et al. ${ }^{27} \mathrm{CVI}=$ cerebrovascular infarction.

\section{DIAGNOSTIC AND RECLASSIFICATION} SPECIFICITY

In the non-multiple sclerosis group $(n=47)$, five patients would have been falsely classified as having probable multiple sclerosis on the basis of the clinical examination alone. This corresponds to a specificity of the clinical examination of $89 \%$. All paraclinical tests yielded abnormal results in some nonmultiple sclerosis patients, and some of these abnormal results would have led to incorrect reclassification of these patients to probable or definite multiple sclerosis (table 4). The number of falsely reclassified patients was greatest for MRI, followed by SSEPs, MEPs, VEPs, and CSF (MRI $v$ CSF, $\mathrm{P}<0 \cdot 05$ ). Application of the criteria of Fazekas et $a l^{27}$ to the analysis of MRIs reduced the number of falsely reclassified patients considerably (table 4). Most of the falsely reclassified patients had cerebrovascular disorders (table 4). The incidence of non-specific MRI abnormalities is greater after the age of $50,{ }^{27}$ and our six falsely reclassified patients were somewhat older (mean 45, range 20-69 years) than the non-multiple sclerosis group as a whole (41 years). Nevertheless, four of the six patients were younger than 50 years, suggesting that false reclassifications by MRI may not only be a problem of older age. These data allowed calculation of diagnostic and reclassification specificities for each of the paraclinical tests (table 4). The reclassification specificity decreased considerably if more than one paraclinical test was considered: multimodal EPs had a reclassification specificity of $87 \%$, and if all paraclinical tests were combined (and the criteria of Fazekas $e t a l^{27}$ were applied to MRI interpretation), the reclassification specificity was only $72 \%$.

\section{Discussion}

Paraclinical tests are performed to increase the certainty of a clinically suspected diagnosis. This is of particular interest in a disease such as multiple sclerosis, for which a specific test does not exist. In this study, the efficacy of paraclinical testing in multiple sclerosis was analysed by focusing on the diagnostic power of various methods in a clinical setting. It shows that mere evaluation of diagnostic sensitivities may be inappropriate. By using a stratified approach in unselected patients with suspected multiple sclerosis we analysed the impact of different paraclinical tests on PCC classification. ${ }^{2}$ As expected, the reclassification sensitivity (the percentage of patients who could be reclassified by an abnormal result) was lower than the diagnostic sensitivity (the percentage of patients with an abnormal result) in all of the performed tests. Because this reclassification index (table 2) differed largely between the tests, comparison of these tests on the basis of diagnostic sensitivity alone may be misleading. When taken as single tests, MRI had the greatest reclassification sensitivity $(60 \%)$, followed by CSF analysis (31\%), and VEPs (28\%), MEPs (19\%), and SSEPs $(12 \%)$.

By including patients with suspected multiple sclerosis in whom subsequently a different diagnosis was made, we were able to calculate specificities of the paraclinical tests for reclassification according to the PCC (table 4). The reclassification specificities were relatively high for all performed tests. It is, however, important to note and of considerable clinical interest that by increasing the number of paraclinical examinations, the number of falsely reclassified non-multiple sclerosis patients increased as well. The diagnostic accuracy in a given patient may thus decrease rather than increase if a great many paraclinical examinations are performed. Although the few non-multiple sclerosis patients in our study did not allow a thorough assessment, they suggest a lower specificity of MRI compared with the other paraclinical tests. The MRI results would have led to an incorrect reclassification to probable or definite multiple sclerosis in six of 47 non-multiple sclerosis patients, even if the restrictions described by Fazekas $e t a l^{27}$ were used for the interpretation. Contrary to MRI, only one patient would have been falsely reclassified by the result of the CSF analysis (table 3). Summarised, our data support our clinical impression that sometimes MRI findings that are highly suggestive of multiple sclerosis fail to exclude other neurological conditions, as stated previously by others. ${ }^{57}$ Hence, the high reclassification sensitivity of the MRI may, to some extent, be at the expense of a comparatively low reclassification specificity; and diagnostic criteria such as those proposed by Fazekas $e t a^{27}$ should be used for the interpretation of MRI abnormalities.

A possible reason for performing paraclinical tests is to confirm and characterise clinically doubtful or suspected findings. This aspect was not assessed in the present investigation, and applies especially to EP studies. For example, in a patient with visual disturbances it may be helpful to perform VEPs, as a prolonged latency supports the presence of an 
optic neuritis, whereas a decreased amplitude of the cortical response may suggest an ophthalmological problem or an axonal lesion to the optic nerve. ${ }^{21}$ Such a patient would not have been reclassified by an abnormal VEP result. Also the diagnostic values of MEPs and SSEPs is probably underrated by our analysis, because they may be helpful in ascertaining doubtful clinical findings such as brisk reflexes without spasticity, equivocal extensor plantar responses, or ambiguous sensory symptoms of multiple sclerosis.

It is difficult to compare our data with previously published studies. Firstly, most investigations only reported diagnostic sensitivities of various paraclinical tests without evaluating the impact of these abnormal results on the diagnostic appraisal. Secondly, the diagnostic sensitivity of paraclinical testing is known to increase during the course of the disease..$^{23} 29$ The average duration of the disease of our cohort of patients was probably shorter than in most previous studies, as we included cases with possible and probable multiple sclerosis. Thirdly, by excluding patients with possible multiple sclerosis who could not yet be classified according to the PCC, previous studies excluded a most important group of patients who only later developed probable or definite multiple sclerosis. We are aware that our inclusion of patients with possible multiple sclerosis with monophasic syndromes bears the risk of misinterpretation of an acute disseminated encephalomyelitis (ADEM) as multiple sclerosis. As ADEM is much less common than multiple sclerosis, this risk is of little clinical relevance, however. In a pathomorphological study, Izquierdo et al ${ }^{30}$ showed that, based on the PCC, the average delay for diagnosing clinically definite multiple sclerosis was longer than eight years. Hence, when assessing the diagnostic efficacy of a test, exclusion of cases with possible multiple sclerosis from the study fails to meet the clinical reality. Finally, the specificity of paraclinical studies to reclassify patients with suspected multiple sclerosis according to the PCC criteria has not been evaluated so far.

The superiority of MRI in our investigation was due to its known high sensitivity in detecting clinically silent lesions. ${ }^{52931}$ Dissemination in space can be directly demonstrated by MRI whereas EPs suggest spatial dissemination only in the context of clinical findings. Paty et al, ${ }^{7}$ analysing a similar patient group ( $n=200)$, found a similarly high diagnostic sensitivity for MRI (62\%) compared with sensitivities between $46 \%$ and $49 \%$ for CSF, VEPs, and SSEPs. In a follow up study of the same patients, ${ }^{32}$ the initial MRI had the greatest predictive value for later evolution to clinically definite multiple sclerosis, followed by CSF/VEPs and SSEPs. Similar results were reported by Filippini $e t$ $a l,{ }^{31}$ who found a diagnostic sensitivity of $70 \%$ for MRI in 34 cases of suspected multiple sclerosis. In these studies, however, the impact of the abnormal test results on the initial diagnostic assessment was not evaluated.

It has been suggested that multimodal EP recordings may increase the diagnostic yield of electrophysiological testing in multiple sclerosis. ${ }^{63}$ This was confirmed in the present study, where multimodal EPs allowed reclassification in a greater number of patients than single EP studies. Even if all three EP modalities were combined, however, MRI had a superior reclassification sensitivity $(60 \%$ for MRI $v 46 \%$ for multimodal EPs; figure). The low reclassification sensitivity of MEPs and SSEPs in our study may be due to our restrictive interpretation based on the Poser committee recommendations. ${ }^{2}$ An abnormal result in EPs was only considered for reclassification if none of the clinical findings could be associated with a lesion along the known EP pathways. This is reflected in the high percentage of abnormal multimodal EP and SSEP results and their relatively low reclassification sensitivity (table 2). Nevertheless, EP testing may be useful in patients in whom the MRI or CSF testing is not diagnostic, as was the case in 18 or 39 of our patients with non-reclassifying MRI or CSF respectively and abnormal EPs (table 3). In 39 patients with suspected multiple sclerosis, Farlow et al ${ }^{29}$ found multiple MRI lesions in $72 \%$, but abnormal multimodal EPs only in $41 \%$. Similarly, Cutler et $a l^{5}$ found abnormal MRIs in 21 of 27 patients with definite or probable multiple sclerosis (78\%), but abnormal multimodal EPs in only 14 of these patients (58\%). By contrast, Giesser $e t a l^{6}$ found a higher sensitivity for multimodal EPs (78\%) than for MRI (65\%) in a group of 23 patients with possible multiple sclerosis. It should be noted that in these studies multimodal EPs included VEPs, SSEPs, and acoustic evoked brainstem potentials.

In our cohort of patients, VEPs had a greater chance to detect subclinical lesions than had MEPs and SSEPs, because fewer patients complained of visual disturbances (12 patients in the multiple sclerosis group). Thus, even though VEP abnormalities were considerably less frequent than MEP or SSEP abnormalities, they led to a greater relative number of reclassifications (table 2). Previous studies have also shown that VEPs may have a superior sensitivity to detect clinically silent lesions ${ }^{22}$ even though their diagnostic sensitivity in multiple sclerosis may be lower than EP modalities. ${ }^{25}$

The present direct comparison of MRI/EPs and CSF may be inappropriate from a theoretical standpoint, because of their different appreciation in the PCC classification. These tests are not directly competitive, because CSF furnishes information about possible CNS inflammation, and MRI/EP about possible dissemination in space. The direct comparison in the present study was chosen from a clinical standpoint, where the diagnostic impact (sensitivity and specificity) of an examination is the main interest and not necessarily the pathophysiological nature of abnormal findings or the way by which they are achieved. Even though CSF analysis had a similar diagnostic sensitivity as MRI, the reclassification sensitivity of CSF was only 
half of that of MRI (table 2). By its special appreciation within the PCC, however, CSF analysis was particularly powerful in allowing reclassification to definite multiple sclerosis (figure). In previous studies, the weight of CSF analysis for the diagnosis of multiple sclerosis has been a contentious issue. Morrisey et $a l^{34}$ found MRI more powerful than CSF in predicting the evolution of clinically isolated syndromes in multiple sclerosis. Also others ${ }^{31}{ }^{35}$ suggested that CSF analysis does not add substantially to diagnostic certainty. In our investigation, however, 12 of 57 patients $(21 \%)$ with normal or non-diagnostic MRI could be reclassified by the CSF finding (table 3). In 90 patients with multiple sclerosis with a cranial MRI not "strongly suggestive of multiple sclerosis", Lee et $a l^{32}$ found 25 patients $(28 \%)$ with positive CSF oligoclonal bands, four of them developing clinically definite multiple sclerosis in the follow up. Sharief and Thompson ${ }^{36}$ found a greater predictive value of CSF (including IgM and IgG analysis) for the later development to definite multiple sclerosis, than MRI. Five of their 45 patients with normal MRI findings showed an evolution to multiple sclerosis after a mean follow up of 18 months.

To our knowledge, only one previous investigation used a similar analysis of reclassification sensitivity as ours. Gilmore et al ${ }^{10}$ analysed the diagnostic value of paraclinical testing in a group of 40 patients with possible or probable multiple sclerosis. The diagnostic reclassification sensitivity of all tests (CSF, MRI, and multimodal EPs) was $63 \%$ in their patients, compared to $78 \%$ in the present study. Analysis of CSF had a reclassification sensitivity of $25 \%$, compared with $31 \%$ in our investigation, and multimodal EPs combined with CSF $53 \%$ ( $59 \%$ in this study). On its own, MRI was indicative of a silent lesion in $45 \%$ only. The findings for MRI and EPs, however, cannot be directly compared with ours, because Gilmore et $a l^{10}$ included the results of the CSF analysis for the baseline classification and subsequently calculated the diagnostic impact of the other paraclinical tests. Also, the performed EPs were different (VEPs, SSEPs, and BAEPs).

In conclusion, our data suggest that the clinical appraisal of suspected multiple sclerosis should start with a cranial MRI and a lumbar puncture for CSF testing. The combination of these two examinations seems particularly useful as they assess two different aspects of the disease-namely, dissemination of lesions in space (MRI) and CNS inflammation (CSF). Moreover, it is the combination of the examinations that yields the greatest specificity (CSF) and the greatest sensitivity (MRI) for reclassifying patients with multiple sclerosis according to the PCC. If MRI and CSF do not allow the attainment of a probable or definite diagnosis (or a lumbar puncture is considered too invasive by the patient), further testing can follow, depending on the clinical situation, by the use of VEPs, MEPs, or SSEPs, or a combination of these with the aim of disclosing clinically silent lesions. Studies on EPs remain helpful especially in evaluating doubtful clinical signs, and, due to their high sensitivity and easy performance, MEPs seem particularly suitable for screening patients with equivocal symptoms. ${ }^{131537}$ One should be aware, however, that performing additional tests may bear the risk of false reclassifications of nonmultiple sclerosis patients to multiple sclerosis. A greater number of paraclinical examinations may thus decrease rather than increase the diagnostic accuracy in multiple sclerosis.

We thank our colleagues of the Department of Neuroradiology (Head: Professor G Schroth) for their collaboration. We also thank Dr J Kesselring (Valens) for helpful comments. This work was supported by the Swiss National Science work was supported by the

1 Schumacher GA, Beebe G, Kibler RF, et al. Problems of experimental trials of therapy in multiple sclerosis: report of the panel on the evaluation of experimental trials of therapy in multiple sclerosis. Ann NY Acad $S_{c i}$ 1965;122:552-68.

2 Poser CM, Paty DW, Scheinberg L, et al. New diagnostic criteria for multiple sclerosis: guidelines for research protocols. Ann Neurol 1983;13:227-31.

3 Tackmann W, Strenge H, Barth R, Sojka RA. Evaluation of various brain structures in multiple sclerosis with multimodality evoked potentials, blink reflex and multimodality evoked potentials, blink
nystagmography. $\mathcal{F}$ Neurol 1980;224:33-46.

4 Walsh JC, Garrick R, Cameron J, McLeod JG. Evoked potential changes in clinically definite multiple sclerosis: a two year follow-up study. $\mathcal{F}$ Neurol Neurosurg Psychiatry 1982;45:494-500

5 Cutler JR, Aminoff MJ, Brant-Zawadzki M. Evaluation of patients with multiple sclerosis by evoked potentials and magnetic resonance imaging: a comparative study. Ann Neurol 1986;20:645-6.

6 Giesser BS, Kurtzberg D, Vaughan HG, et al. Trimodal evoked potentials compared with magnetic resonance imaging in the diagnosis of multiple sclerosis. Arch Neurol 1987;44:281-4.

7 Paty DW, Oger JJF, Kastrukoff LF, et al. MRI in the diagnosis of multiple sclerosis: a prospective study with comparison of clinical evaluation, evoked potentials, parison of clinical evaluation, evoked potentials,
oligoclonal banding, and CT. Neurology 1988;38:180-5.

oligoclonal banding, and CT. Neurology 1988;38:180-5.
8 Jones SM, Streletz LJ, Raab VE, Knobler RL, Lublin FD. Lower extremity motor evoked potentials in multiple sclerosis. Arch Neurol 1991;48:944-8.

9 Mayr N, Baumgartner C, Zeitlhofer J, Deeke L. The sensitivity of transcranial cortical magnetic stimulation in detecting pyramidal tract lesions in clinically definite multiple sclerosis. Neurology 1991;41:566-9.

10 Gilmore RL, Kasarskis EJ, Carr WA, Norvell E. Comparative impact of paraclinical studies in establishing the diagnosis of multiple sclerosis. Electroenceph Clin Neurophysiol 1989;73:433-42.

11 O'Connor P, Tansey C, Kucharczyk W, et al. A randomized trial of test result sequencing in patients with suspected multiple sclerosis. Arch Neurol 1994;51:53-9.

12 Rossini PM, Zarola F, Floris R, et al. Sensory (VEP, BAEP, SEP) and motor-evoked potentials, liquoral and magnetic resonance findings in multiple sclerosis. Eur Neurol 1989;29:41-7.

13 Eisen AA, Shtybel W. AAEM minimonograph No35: clinical experience with transcranial magnetic stimulation. Muscle Nerve 1990;13:995-1011.

14 Hess CW, Mills KR, Murray NMF. Measurement of central motor conduction in multiple sclerosis using magnetic brain stimulation. Lancet 1986;ii:355-8.

15 Hess CW, Mills KR, Murray NMF, Schriefer TN. Magnetic brain stimulation: central motor conduction studies in multiple sclerosis. Ann Neurol 1987;22: 744-52.

16 Rösler K, Mathis J, Hess CW. Diagnosis of multiple sclerosis (MS): does magnetic cortex stimulation affect Poser criteria? Electroenceph Clin Neurophysiol 1993;86: 24P.

17 Mehta PD, Patrick BA. Detection of oligocloncal bands in unconcentrated CSF: isoelectric focusing and silver unconcentrated CSF: isoelectric

18 Schipper HI, Kruse H, Reiber H. Silver-staining of oligoclonal IgG subfractions in cerebrospinal fluid after isoelectric focusing in thin-layer polyacrylamide gels. Science tools 1984;31:5-6.

19 Tibbling G, Link H, Ohmann S. Principles of albumin and IgG analysis in neurological disorders. I. Establishment of reference values. Scand $\mathcal{F}$ Clin Lab Invest 1977;37: 385-90.

20 Reiber H. Eine aktuelle Darstellung des LiquorproteinProfils zur Differentialdiagnose von Schrankenfunktionsstörungen und entzündlichen Prozessen des Zentralnervensystems. Akt Neurol 1980;7:127-34. 
21 Stöhr M, Dichgans J, Diener HC, Buettner UW. Evozierte Potentiale. SEP-VEP-AEP-EKP-MEP. Berlin: Springer Verlag, 1989.

22 Polman CH, Koetsier JC, Wolters EC. Multiple sclerosis: incorporation of results of laboratory techniques in the diagnosis. Clin Neurol Neurosurg 1985;87:187-92.

23 Comi C, Martinelli V, Medaglini S, et al. Correlation between multimodal evoked potentials and magnetic resonance imaging in multiple sclerosis. $\mathcal{f}$ Neurol 1989; 236:4-8.

24 Baumhefner RW, Tourtellotte WW, Syndulko K, et al. Quantitative multiple sclerosis plaque assessment with magnetic resonance imaging. Its correlation with clinical marameters, evoked potentials, and intra-blood-brain paramers, evis. Arch Neurol 1990;47:19-26.

25 Ravnborg M, Liguori R, Christiansen P, Larsson H, Soelberg Sorensen $\mathbf{P}$. The diagnostic reliability of magnetically evoked motor potentials in multiple sclerosis. netically evoked motor potentials

26 Rösler KM, Hess CW, Heckmann R, Ludin HP. Significance of shape and size of the stimulating coil in magnetic stimulation of the human motor cortex. Neurosci Lett 1989;100:347-52.

27 Fazekas F, Offenbacher H, Fuchs S, et al. Criteria for an increased specificity of MRI interpretation in elderly subjects with suspected multiple sclerosis. Neurology 1988;38:1822-5.

28 Offenbacher H, Fazekas F, Schmidt R, et al. Assessment of MRI criteria for a diagnosis of multiple sclerosis. Neurology 1993;43:905-9.

29 Farlow MR, Markand ON, Edwards MK, Stevens JC, Kolar OJ. Multiple sclerosis: magnetic resonance imag- ing, evoked responses, and spinal fluid electrophoresis. Neurology 1986;36:828-31.

30 Izquierdo G, Hauw J-J, Lyon-Caen O, et al. Value of multiple sclerosis diagnostic criteria. 70 autopsy-confirmed tiple sclerosis diagnostic criteria. Arch Neurol 1985;42:848-50.

31 Filippini G, Comi GC, Cosi V, et al. Sensitivities and predictive values of paraclinical tests for diagnosing multiple sclerosis. F Neurol 1994;241:132-7.

32 Lee KH, Hashimoto SA, Hooge JP, et al. Magnetic resonance imaging of the head in the diagnosis of multiple sclerosis: a prospective 2-year follow-up with comparison of clinical evaluation, evoked potentials, oligoclonal banding, and CT. Neurology 1991;41:657-60.

33 Chiappa KH. Pattern shift visual, brainstem auditory, and short-latency somatosensory evoked potentials in multiple sclerosis. Neurology 1980;30:110-23.

34 Morrisey SP, Miller DH, Kendall BE, et al. The significance of brain magnetic resonance imaging abnormalities at presentation with clinically isolated syndromes suggestive of multiple sclerosis. Brain 1993;116:135-46.

35 Giang DW, Grow VM, Mooney C, et al. Clinical diagnosis of multiple sclerosis. The impact of magnetic resonance of multiple sclerosis. The impact of magnetic resonance 61-66.

36 Sharief MK, Thompson EJ. The predictive value of intrathecal immunoglobulin synthesis and magnetic resonance imaging in acute isolated syndromes for subsequent development of multiple sclerosis. Ann Neurol 1991;29:147-51.

37 Murray NMF. The clinical usefulness of magnetic cortical stimulation. Electroenceph Clin Neurophysiol 1992;85: $81-5$. 